\title{
Modifications précoces de la composition en acides gras des cardiolipides et autres phospholipides de mitochondries de foie de rat lors d'une carence alimentaire en acides gras essentiels suivie d'une réplétion
}

\author{
R. L. WOLFF
}

Unité de Biochimie-Biotechnologie de I'I.T.E.R.G., Laboratoire de Lipochimie Alimentaire Département Alimentation et Nutrition. Université de Bordeaux 1 Avenue des Facultés, 33405 Talence Cedex, France.

Summary. Early modifications in the fatty acid profile of cardiolipin and other phospholipids from rat liver mitochondria during the onset of essential fatty acid deficiency and its reversal.

Weaned rats (21-day old, $44 \pm 2 \mathrm{~g}$ ) were distributed into 3 groups. The first group was raised on a laboratory diet for 7 or 20 days (control group). The second was fed a diet containing $0.07 \%$ fat for $1,2,3,7$ or 66 days. The third one was fed the low-fat diet for 7 days and then switched to a laboratory chow diet for $1,2,5$ or 9 days. Cardiolipin (CL) on the one hand and other mitochondrial phospholipids taken as a whole (PLm) on the other hand were prepared from liver mitochondria and their fatty acids analysed.

Polyunsaturated fatty acids in PLm (18:2 (n-6), 20:4 (n-6), $22: 6$ (n-3) acids) decreased abruptly during the first 3 days of fat deficiency and then remained rather stable till day 7 . CL behaved in a quite different way. $18: 2(n-6)$ acid, the major polyunsaturated fatty acid of CL, decreased continuously between day 1 and day 7 from $79 \%$ to $33 \%$. A value of $19.6 \%$ was reached on day 66 . When deficient rats were transferred to an equilibrated diet, the fatty acid profile of PLm was rapidly restored. Major effects were already achieved during the first $24 \mathrm{~h}$ and a fatty acid composition identical to that of control rats was reached within 2 days. A considerably longer period (about 9 days) was necessary for $C L$ to reach a level analogous to that of control rats.

The diminution of linoleic acid in $\mathrm{CL}$ was brought about by increases in the levels of monoenoic acids : palmitoleic $(16: 1(n-7))$, oleic $(18: 1(n-9))$ and cis-vaccenic $(18: 1$ $(n-7))$ acids which accounted for $18.0 \%, 17.7 \%$ and $17.9 \%$ respectively on day 7 . Increases in the (n-7) monoenes remained comparatively low in PLm, which showed a sharp rise in their oleic acid content (from $3.9 \%$ to $12.7 \%$ in 3 days). The proportion of cis-vaccenic acid relative to total octadecenoic acids was decreased from $75 \%$ to $50 \%$ in $\mathrm{CL}$. A similar trend, but of smaller magnitude, was also noticed in PLm. The endogenously synthesized $18: 2(n-7)$ and $20: 3(n-9)$ acids were unevenly distributed between CL and PLm. 18: $2(n-7)$ acid was preferentially esterified in $C L$ where it accounted for $1.5 \%$ on day 7 of fat deficiency and for $4.3 \%$ on day $66(0.7 \%$ and $2 \%$ in PLm). On the contrary, $20: 3(n-9)$ acid was almost exclusively incorporated in PLm : $4.3 \%$ after 7 days and $13 \%$ after 66 days. Corresponding values for $\mathrm{CL}$ were $<0.1 \%$ and $0.8 \%$. Elimination of these biochemical markers of deficiency was rather slow either in CL or in PLm.

Fat deficiency induced a slight but significant increase in the level of palmitic acid in $C L$ and in PLm during the first 3 days. In CL, this effect was reversed less than 2 days after transfer to an equilibrated diet, but a significant drop below the level of control animals 
occurred in PLm. Stearic acid remained unchanged in CL (mean value : $1 \%$ ) but varied in an opposite direction to that of palmitic acid in PLm.

\section{Introduction.}

Chez les animaux, les cardiolipides (CL), ou diphosphatidylglycérols, sont synthétisés exclusivement dans la membrane interne des mitochondries (McMurray et Jarvis, 1979) où ils restent essentiellement localisés. Dans les cas du cœur et du foie de rat, ils représentent 15 à $20 \%$ du phosphore lipidique total de ces membranes (Colbeau et al., 1971 ; Wolff et al., 1984). Les CL sont associés à de nombreuses enzymes de la membrane mitochondriale interne (pour une revue, $c f$. loannou et Golding, 1979), et en particulier à celles liées à la production d'ATP : ATPase (Santiago et al., 1973), NADH-ubiquinone réductase (Fry et Green, 1981), cytochrome-oxydase (Merle et Kadenbach, 1982), protéine transporteuse d'ADP/ATP (Beyer et Klingenberg, 1985).

Les $C L$ de différents organes de rats adultes normaux, à l'exception de ceux du cerveau (Yamaoka et al., 1988), contiennent de 60 à $90 \%$ ou plus d'acide linoléique [18:2 (n-6)] (Yurkowski et Walker, 1970 ; Bruce, 1974 ; Palmer et al., 1981 ; Wolff et al., 1984). Les autres acides gras majeurs sont d'ordinaire les acides oléique [18:1 (n-9)] et cis-vaccénique [18:1 (n-7)], ce dernier étant souvent l'isomère principal (Patton et al., 1982; Wolff et al., $1985 \mathrm{a}$, b ; Wood et al., 1986). II est à noter que les CL, contrairement aux autres phospholipides, n'incorporent pas les isomères trans $\mathrm{d}$ 'acides octadécénoïques (Blomstrand et Svensson, 1983 ; Wolff et al., 1984 ; Astorg et Chevalier, 1987) ni l'isomère trans, trans de l'acide linoléique (Combe et al., 1980). Les CL se distinguent aussi des autres phospholipides par leur teneur en acides gras saturés qui ne dépasse généralement guère 6 ou $8 \%$ des acides gras totaux (Getz et Bartley, 1959; Palmer et al., 1981 ; Patton et al., 1982 ; Wolff et al., 1984, 1985 a, b ; Yamaoka et al., 1988).

De nombreuses études ont été menées, chez le rat, sur les effets d'une déficience alimentaire en matière grasse ou en acide linoléique sur les compositions en acides gras des lipides, mais peu d'entre elles font mention des CL (Bartley, 1964 ; Biran et al., 1965 ; Tischer et Glenn, 1965 ; Yamamoto et al., 1965 ; Johnson et Ito, 1965 ; Stancliff et al., 1969 ; Yurkowski et Walker, 1970 ; Wood, 1975 ; Divakaran et Venkataraman, 1977 ; Ishinaga et al., 1982 ; Wolff et al., 1985 a). Dans ces études, les rats ont été nourris avec des régimes carencés pendant des périodes assez longues, et, à notre connaissance, jamais inférieures à une semaine. Or, des résultats préliminaires nous ont montré que l'essentiel des modifications de la composition en acides gras des CL du foie de rat apparaissait pendant cette période. En raison de l'importance quantitative et fonctionnelle des $\mathrm{CL}$, de leur origine métabolique distincte de celle des autres phospholipides et de leur affinité particulière pour l'acide linoléique, nous avons voulu suivre les événements précoces de l'instauration d'une déficience en matière grasse sur la composition en acides gras des $\mathrm{CL}$, puis de la restauration de cette composition 
lorsque les animaux étaient à nouveau nourris avec un régime équilibré. Parallèlement, nous avons suivi l'évolution de la composition en acides gras des autres phospholipides mitochondriaux (PLm) dont la synthèse de novo est surtout d'origine microsomale (Van den Bosch, 1974).

\section{Matériels et méthodes.}

Animaux et régimes. - Quarante-deux rats mâles de souche Wistar, pris au sevrage (21 jours, $44 \pm 2 \mathrm{~g}$ ), ont été nourris 1 jour avec un aliment complet (A-04, UAR, Villemoisson-sur-Orge, France) renfermant $4 \%$ en poids de matière grasse, dont $50 \%$ et $3,7 \%$ des acides gras étaient, respectivement, de l'acide linoléique et de l'acide linolénique. Trente-huit de ces rats ont ensuite été nourris avec un aliment contenant $0,07 \%$ en poids de lipides (UAR) dont $60 \%$ des acides gras étaient de l'acide linoléique et $4 \%$ de l'acide linolénique (régime à teneur réduite en lipides: régime $T R L$ ), et le même jour, 4 rats ont été sacrifiés (temps 0 des régimes). Puis, par groupes de 4 ( 6 pour le dernier temps), les rats ont été sacrifiés aux temps 1, 2, 3, 7 et 66 jours. Au temps 7 jours, 16 rats ont été remis à un régime équilibré, et, par groupes de 4, sacrifiés aux temps 8, 9, 12 et 16 jours comptés à partir du temps 0 (régime de réplétion). Huit rats supplémentaires ont été nourris avec le régime équilibré pendant 7 jours pour 4 d'entre eux et pendant 20 jours pour les 4 autres (régime témoin). Les aliments et l'eau étaient fournis ad libitum.

Préparation des mitochondries et de leurs lipides. - Aux temps indiqués, entre 9 et $10 \mathrm{~h}$, les rats ont été décapités après anesthésie légère par l'oxyde d'éthyle, et leur foie a été prélevé. Après rinçage avec une solution tampon (10 mM Tris, $0,25 \mathrm{M}$ sucrose, $2 \mathrm{mM}$ EDTA, $\mathrm{pH} 7,2$ ), les organes rassemblés de 4 rats ont été coupés en morceaux, rincés, et homogénéisés dans un homogénéiseur de Potter avec un volume de tampon égal à 8 fois le poids d'organes ( 2 allers et retours à $600 \mathrm{t} / \mathrm{min}$, puis 2 autres à 800 puis $1000 \mathrm{t} / \mathrm{min}$ ). Après élimination des débris cellulaires par une première centrifugation de $10 \mathrm{~min}$ à $600 \times \mathrm{g}$, les mitochondries ont été récoltées à partir du surnageant par une seconde centrifugation de $10 \mathrm{~min}$ à $6000 \times \mathrm{g}$. Le culot de mitochondries a été rincé 2 fois. Toutes ces opérations ont été effectuées à $4{ }^{\circ} \mathrm{C}$.

A 1 volume de suspension de mitochondries ont été ajoutés successivement 7 volumes de méthanol puis 14 volumes de chloroforme. L'ensemble a été agité pendant $30 \mathrm{~min}$ puis filtré sur un disque en fibres de verre (Whatman GF/C). Les solvants ont été évaporés sous pression réduite, et les lipides, repris dans du chloroforme pur, ont été transférés dans un tube et filtrés. Après évaporation du solvant sous $\mathrm{N}_{2}$, les lipides ont été dissous dans du chloroforme/méthanol (2/1, V/V) contenant $5 \%$ d'eau et lavés selon Folch et al. (1957). Après homogénéisation par du méthanol, la phase inférieure a été évaporée sous $\mathrm{N}_{2}$ et les lipides dissous dans du chloroforme/méthanol $(2 / 1, \mathrm{~V} / \mathrm{V})$ contenant $0,02 \%(\mathrm{Pds} / \mathrm{V}) \mathrm{de}$ 2,6-di-tert-butyl-4-méthylphénol (BHT). Les solutions de lipides ont été conservées à $-20^{\circ} \mathrm{C}$. 
Séparation des CL et des autres phospholipides. - Les CL ont été séparés des autres phospholipides et des lipides neutres par chromatographie sur couche mince (CCM) de gel de silice (Kieselgel H 60, Merck, Darmstadt, RFA) en utilisant I'un après l'autre, dans la même direction, les mélanges oxyde d'éthyle/acétone $(60 / 20, \mathrm{~V} / \mathrm{V})$ et chloroforme/méthanol $(85 / 15, \mathrm{~V} / \mathrm{V})$, contenant tous deux $0,02 \%$ de $\mathrm{BHT}$. Les lipides neutres migrent au front du premier solvant, et les phospholipides restent près de la ligne de dépôt $\left(0<R_{f}<0,2\right)$. Les $C L$ ont un $R_{f}$ de 0,7 environ.

Analyse des acides gras. - Une solution de dichloro-2', $7^{\prime}$-fluorescéine à $0,2 \%$ $(\mathrm{Pds} / \mathrm{V})$ dans l'éthanol à $96^{\circ}$ a été vaporisée sur les plaques de CCM et celles-ci ont été observées sous lumière UV $(254 \mathrm{~nm})$. Les bandes de gel renfermant les $\mathrm{CL}$ d'une part, et l'ensemble des autres phospholipides d'autre part, ont été grattées et placées dans des tubes. $0,8 \mathrm{ml}$ de $\mathrm{BF}_{3}$ méthanolique $(10 \%$, Pds $/ \mathrm{V}$; Flucka, Buchs, Suisse) ont été ajoutés, et les tubes fermés hermétiquement ont été placés à $90^{\circ} \mathrm{C}$ pendant $20 \mathrm{~min}$ (Morrison et Smith, 1964). Les esters méthyliques (EM) d'acides gras ont été analysés par chromatographie en phase gazeuse (CPG) à l'aide d'un appareil Carlo Erba HRGC 4160 équipé d'un détecteur à ionisation de flamme. Les EM ont été séparés dans une colonne WCOT (longueur: $50 \mathrm{~m}$; diamètre intérieur: $0,22 \mathrm{~mm}$ ) de silice fondue CP Wax $52 \mathrm{CB}$ (Chrompack, Midelburg, Hollande). L'injecteur (mode diviseur, rapport 1/40) et le détecteur étaient à $250{ }^{\circ} \mathrm{C}$ et la colonne à $200^{\circ} \mathrm{C}$. La pression du gaz vecteur $\left(\mathrm{H}_{2}\right)$ était de 2 bar. Les aires des pics et leurs pourcentages ont été calculés à l'aide d'un intégrateur Shimadzu ICR-1B. Les EM ont été identifiés selon des méthodes décrites précédemment (Wolff et al., 1985 a).

Les valeurs indiquées dans cette étude sont les moyennes des résultats d'analyses en CPG des EM obtenus par transméthylation de 3 échantillons de CL ou d'autres phospholipides, isolés de façon indépendante à partir des lipides totaux extraits des mitochondries de foies rassemblés de 4 rats. Par commodité de représentation graphique, les valeurs concernant les acides gras des PLm et des $C L$ au temps 66 jours du régime TRL ne sont pas indiquées sur les figures, mais uniquement dans le texte.

\section{Résultats.}

\section{Acide linoléique et acides gras dérivés (série $\mathrm{n}-6$ ).}

Lorsque les rats sont nourris avec le régime TRL, l'acide linoléique diminue immédiatement dans les PLm (fig. 1) : en $24 \mathrm{~h}$, il est réduit de $8,9 \%$ à $5,4 \%$ et, après $48 \mathrm{~h}$, à $2,8 \%$. Ce taux est peu modifié par la suite : $2,2 \%$ au temps 7 jours et $2,3 \%$ au temps 66 jours. La proportion d'acide linoléique dans les PLm est donc rapidement stabilisée. Dans les $C L$, les premiers effets nets ne se font sentir qu'après $24 \mathrm{~h}$ (fig. 1). Entre les jours 0 et 1, une baisse d'acide linoléique de 4 à $5 \%$ est enregistrée, mais entre les jours 1 et 2, cette baisse est de $20 \%$. Contrairement à ce qui se produit dans les PLm, cette diminution se poursuit dans 
les $C L$ : I'acide linoléique représente $33 \%$ des acides gras de ce phospholipide au temps 7 jours, et $19 \%$ au temps 66 jours. Après passage à un régime équilibré, le taux normal d'acide linoléique dans les PLm (c'est-à-dire un taux identique à celui des PLm de rats du lot témoin) est rétabli en 24 à $48 \mathrm{~h}$ (fig. 1). Dans les $C L$, cet état n'est retrouvé qu'après 9 jours.

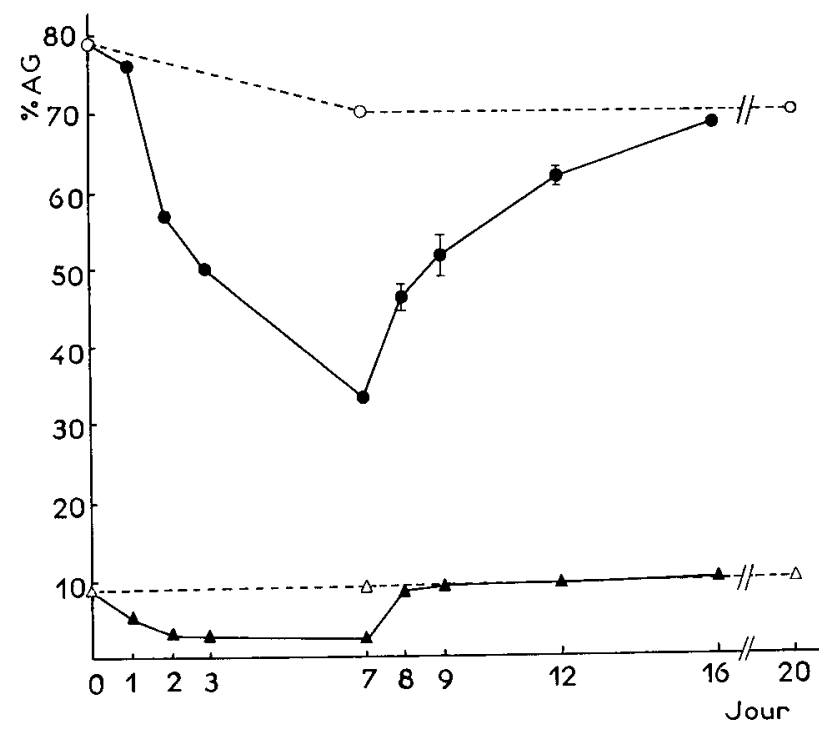

FIG. 1. - Evolution, au cours du temps, de la teneur en acide $18: 2(n-6)$ des CL (- - - et autres phospholipides ( $\mathbf{\Delta} \mathbf{\Delta})$ de mitochondries de foie de rats nourris avec un régime $T R L$ pendant 7 jours puis un régime équilibré pendant 9 jours, ou nourris pendant 20 jours avec un régime équilibré ( $\mathrm{CL}: \bigcirc--\bigcirc$; autres phospholipides : $\triangle--\triangle$ ). \% AG : pourcentage des surfaces d'EM d'acides gras totaux de la fraction considérée. Jour : jour du sacrifice des rats, compté à partir du début du régime TRL. Chaque point représente la moyenne de 3 valeurs, obtenues par analyse en CPG des EM préparés par transméthylation de 3 échantillons de CL ou d'autres phospholipides, ceux-ci étant isolés de façon indépendante à partir des lipides totaux extraits des mitochondries de foies rassemblés de 4 rats. Lorsque les déviations standards ne sont pas indiquées, elles sont égales ou inférieures à la taille des symboles.

La somme des pourcentages des autres acides gras de la série ( $n-6)$ (acides $20: 2,20: 3,20: 4$ ) varie peu dans les CL (fig. 2). Dans le détail (résultats non montrés), on note, chez les rats du lot TRL, une baisse de l'acide $20: 2(n-6)$ de $1,9 \%$ au temps 0 à $0,7 \%$ au temps 7 jours. Neuf jours après transfert sur régime équilibré, son taux remonte à $1,2 \%$, mais reste inférieur à celui déterminé chez les rats du lot témoin : $1,7 \%$. Les acides $20: 3(n-6)$ et $20: 4(n-6)$, représentant tous deux $0,9 \%$ des acides gras des $C L$ au temps 0 , augmentent pour atteindre, respectivement, 2,6 et $1,6 \%$ au temps 7 jours du régime TRL $(4,6$ et $1,8 \%$ au temps 66 jours). Après transfert des rats sur régime équilibré, ces valeurs décroissent jusqu'à $1,9 \%$ et $1,3 \%$. Par contre, dans les PLm, où l'acide 
arachidonique est le principal dérivé de l'acide linoléique $(18,8 \%$ au temps 0$)$, une baisse importante des acides $(n-6)$ se produit pendant les 3 premiers jours pour se stabiliser ensuite, et atteindre une valeur de $17 \%$ (dont $14,4 \%$ pour le seul acide $20: 4(n-6))$ après 7 jours, valeur peu différente de celle déterminée dans les PLm au temps 66 jours (16\%). Après transfert des animaux sur régime équilibré, les acides gras dérivés de l'acide linoléique retrouvent un taux normal en moins de 2 jours (fig. 2); par la suite, ce taux demeure légèrement supérieur à celui des $\mathrm{PLm}$ de rats nourris depuis le sevrage avec un régime équilibré.



FIG. 2. - Evolution, au cours du temps, de la teneur en acides gras de la série ( $n-6)$ autres que l'acide linoléique, des CL et autres phospholipides de mitochondries de foie de rat. Conditions expérimentales et symboles: voir légende de la figure 1.

\section{Acides gras mono-insaturés.}

Acide palmitoléique [16:1 (n-7)]. - L'acide palmitoléique, constituant mineur des PLm et des $C L$ au temps $0(0,5 \%$ et $0,8 \%$ respectivement), augmente considérablement chez les rats du lot TRL (fig. 3 ). II atteint son taux maximum $(4,7 \%)$ dans les $\mathrm{PLm}$ dès le troisième jour, alors que son augmentation continue jusqu'au septième jour dans les CL pour atteindre $18 \%$. Au temps 66 jours, l'acide palmitoléique représente $21 \%$ des acides gras des $C L$, mais il ne dépasse pas $4 \%$ dans les PLm. Le retour à l'état normal après transfert sur régime de réplétion s'effectue en moins de 2 jours dans les PLm, mais en plus de 9 jours dans les CL. 


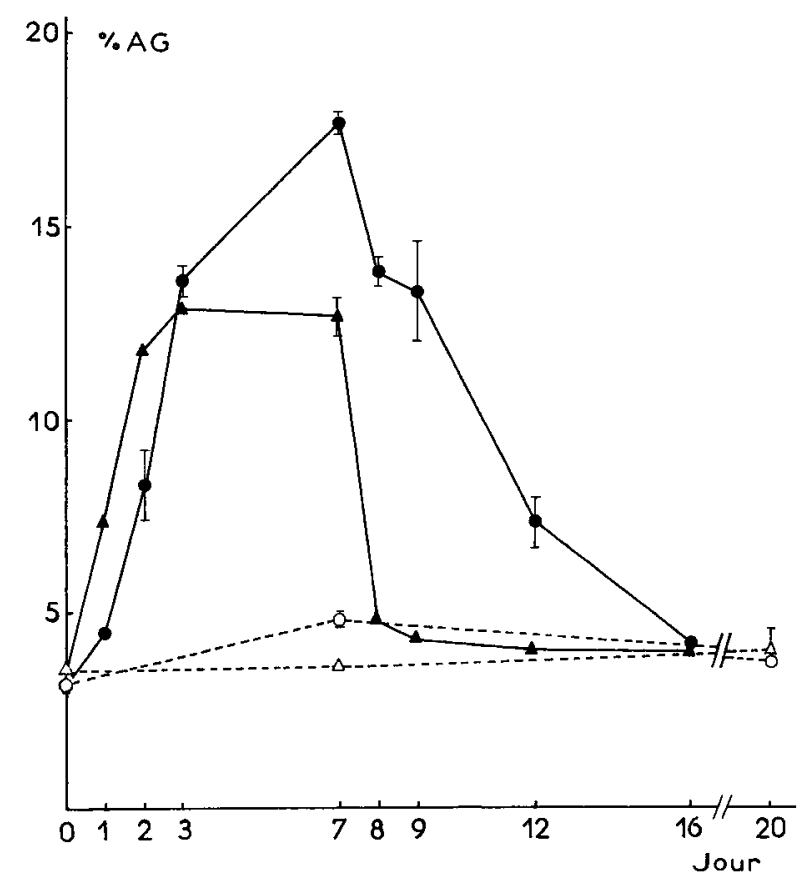

FIG. 3. - Evolution, au cours du temps, de la teneur en acide $16: 1$ (n-7) des CL et autres phospholipides de mitochondries de foie de rats. Conditions expérimentales et symboles: voir légende de la figure 1.

Acide oléique. - Au jour 0 , l'acide oléique représente environ $3,5 \%$ des acides gras des PLm et des CL (fig. 4). Pour ces derniers, l'allure des modifications du taux d'acide oléique est comparable à celle du taux d'acide palmitoléique ; l'augmentation de l'acide oléique se poursuit jusqu'au temps 7 jours pour atteindre $18 \%$ des acides gras totaux, et $19,8 \%$ après 66 jours. Dans les PLm, un maximum de $13 \%$ d'acide oléique est atteint en 3 jours, et maintenu pendant les 4 jours suivants. Mais au temps 66 jours, le taux d'acide oléique est réduit à 9,5\%. Lorsque les animaux sont nourris avec le régime de réplétion, une baisse considérable de l'acide oléique se produit dans les PLm : en $24 \mathrm{~h}$, cet acide chute de $13 \%$ à $4,5 \%$, valeur voisine de celle de rats contrôles (4\%). Par contre, l'acide oléique ne retrouve son taux normal dans les CL que 9 jours environ après le transfert.

Acide cis-vaccénique. - L'acide cis-vaccénique, second acide gras majeur dans les $C L$ au temps 0 , augmente modérément le premier jour sur régime $T R L$, puis plus rapidement pour atteindre $18 \%$ au temps 7 jours (fig. 5). Comme pour les deux autres acides monoénoïques, 9 jours sur régime équilibré sont nécessaires pour que soit rétabli un taux normal d'acide cis-vaccénique. Dans les PLm, cet acide gras augmente jusqu'au temps 3 jours puis se stabilise aux alentours de

Reproduction, Nutrition, Développement, $n^{\circ} 6 \mathrm{~A} / 88-8$ 


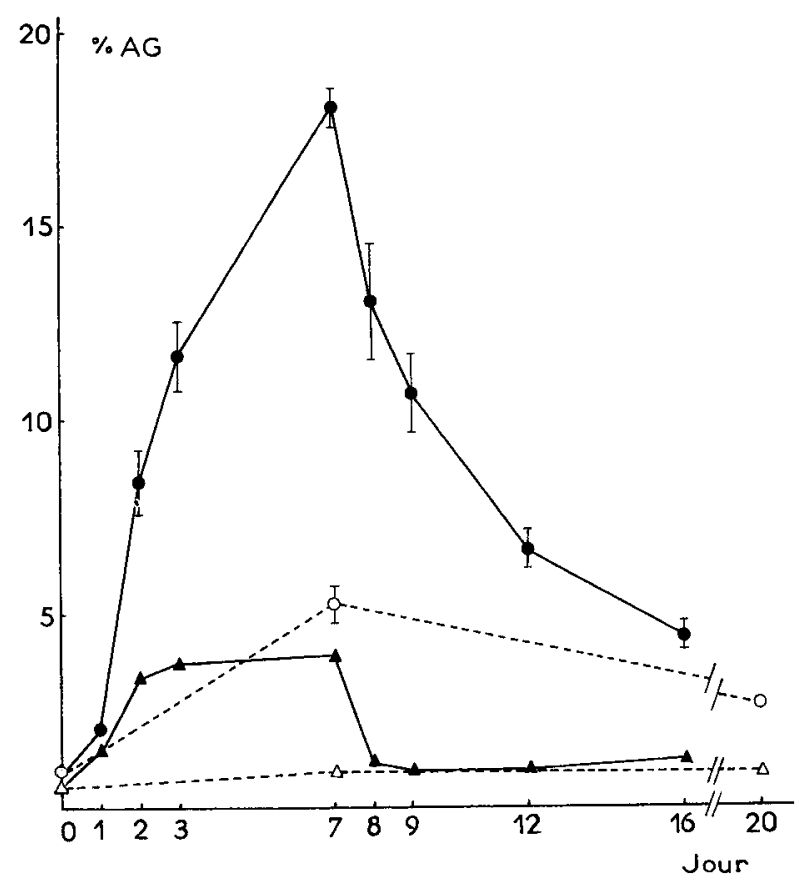

FIG. 4. - Evolution, au cours du temps, de la teneur en acide 18:1 (n-9) des CL et autres phospholipides de mitochondries de foie de rats. Conditions expérimentales et symboles : voir légende de la figure 1.

$4,5 \%$. Le taux normal est rétabli en deux jours après retour à un régime équilibré. Si les rats sont maintenus sur régime $T R L$, le taux d'acide cis-vaccénique reste constant dans les CL $(18,2 \%$ à 66 jours) et dans les PLm $(5,2 \%)$.

Sur régime TRL, les proportions relatives des deux acides octadécénoïques varient largement dans les $C L$. Alors que l'acide cis-vaccénique représente près de $75 \%$ des acides octadécénoïques au temps 0 , sa proportion est réduite à environ $50 \%$ dès le troisième jour, et maintenue à ce taux jusqu'à 66 jours. Après passage sur régime de réplétion, les proportions d'acides oléique et cis-vaccénique retrouvent une valeur normale au bout de 9 jours. Dans les PLm, c'est l'acide oléique qui est l'acide octadécénoïque majeur au temps 0 (70\%). Sur régime TRL, cette proportion est peu affectée et ne dépasse pas $75 \%$. L'état normal est rétabli 1 jour environ après retour au régime équilibré.

\section{Acides gras polyinsaturés d'origine endogène.}

Chez les rats du groupe $T R L$, deux acides polyinsaturés endogènes, les acides $18: 2(n-7)$ et $20: 3(n-9)$, apparaissent dans les PLm des rats du lot TRL (fig. 6). Au temps 3 jours, un état apparemment stationnaire est atteint : les acides $18: 2$ 


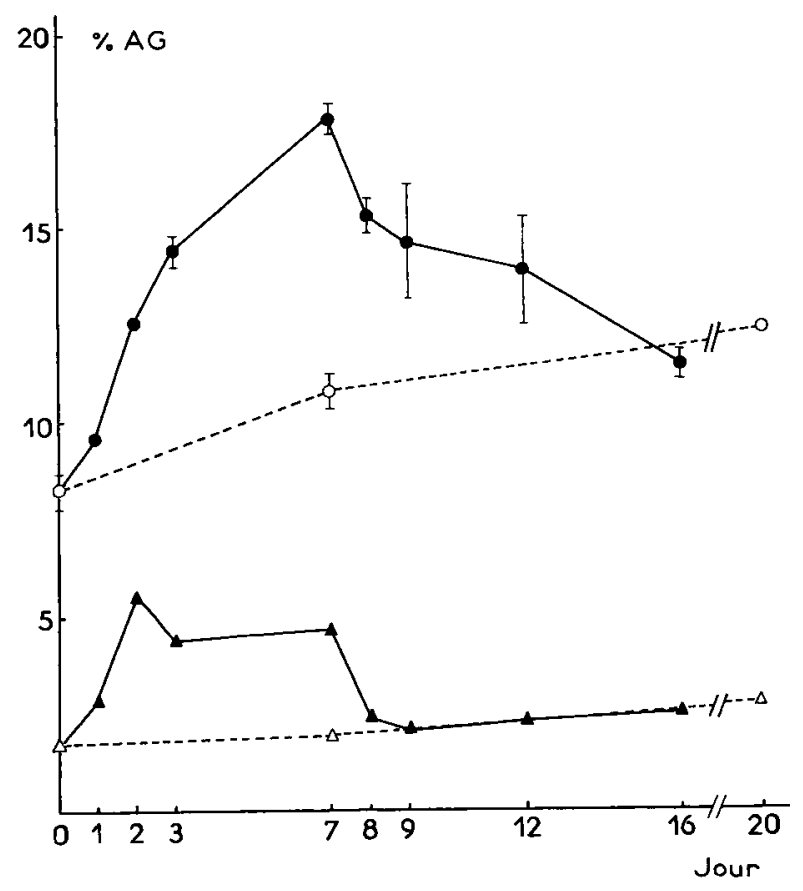

FIG. 5. - Evolution, au cours du temps, de la teneur en acide 18:1 (n-7) des CL et autres phospholipides de mitochondries de foie de rats. Conditions expérimentales et symboles: voir légende de la figure 1.

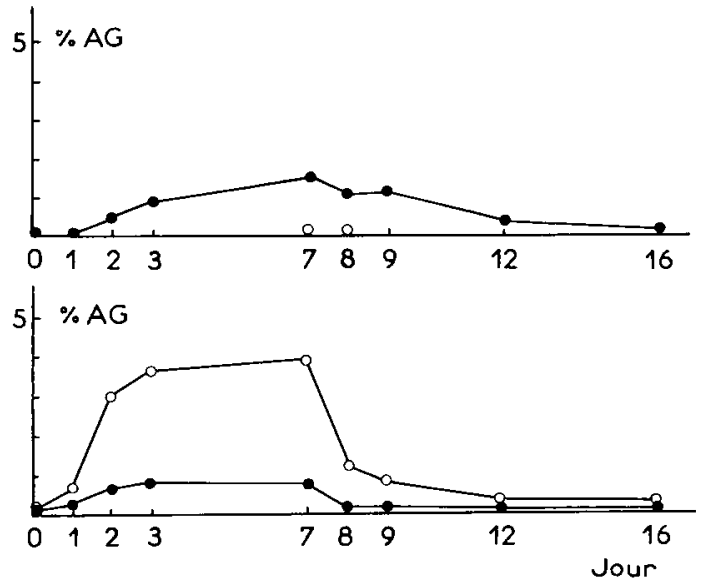

FIG. 6. - Evolution, au cours du temps, de la teneur en acides $20: 3(n-9)$ $(\mathrm{O}-\mathrm{O})$ et $18: 2 \quad(n-7)$ (-) des $C L$ (fig. du haut) et autres phospholipides (fig. du bas) de mitochondries de foie de rats. Conditions expérimentales: voir légende de la figure 1. 
$(n-7)$ et $20: 3(n-9)$ représentent alors $0,7 \%$ et $4 \%$, respectivement, des acides gras. A 66 jours, l'acide $18: 2(n-7)$ n'a pratiquement pas évolué $(0,9 \%)$ alors que l'acide $20: 3(n-9)$ représente, à ce moment, $13 \%$ des acides gras. Dans les CL de foie de rats du lot TRL, l'acide $20: 3(n-9)$ n'est détecté que le septième jour et ne représente, après 66 jours, que $0,8 \%$ des acides gras totaux. Par contre, I'acide $18: 2(n-7)$, qui apparaît le deuxième jour, augmente plus rapidement : il représente $1,5 \%$ au temps 7 jours et $4,3 \%$ au temps 66 jours. La disparition de ces acides gras, tant dans les CL que dans les PLm, est relativement lente: des traces d'acides $18: 2(n-7)$ et $20: 3(n-9)$ sont détectables 9 jours après transfert des animaux sur régime équilibré.

\section{Acides gras saturés.}

Dans les $C L$ et les PLm des rats du groupe TRL, l'acide palmitique augmente d'environ 2,5\% pendant les 3 premiers jours, puis se stabilise jusqu'au temps 7 jours (fig. 7). Après passage sur régime équilibré, cet acide retrouve un taux normal en 1 à 2 jours dans les CL, mais il descend largement en dessous de ce taux dans les PLm pour ne le retrouver qu'entre 5 à 7 jours après le transfert. L'acide stéarique a un comportement inverse de celui de l'acide palmitique dans les PLm, mais dans les $\mathrm{CL}$, son taux reste constant (1\% environ), comme dans les $\mathrm{CL}$ des rats témoins. Globalement, chez les rats du groupe TRL, l'ensemble des acides gras saturés augmente légèrement dans les $C L$, mais il diminue dans les PLm.

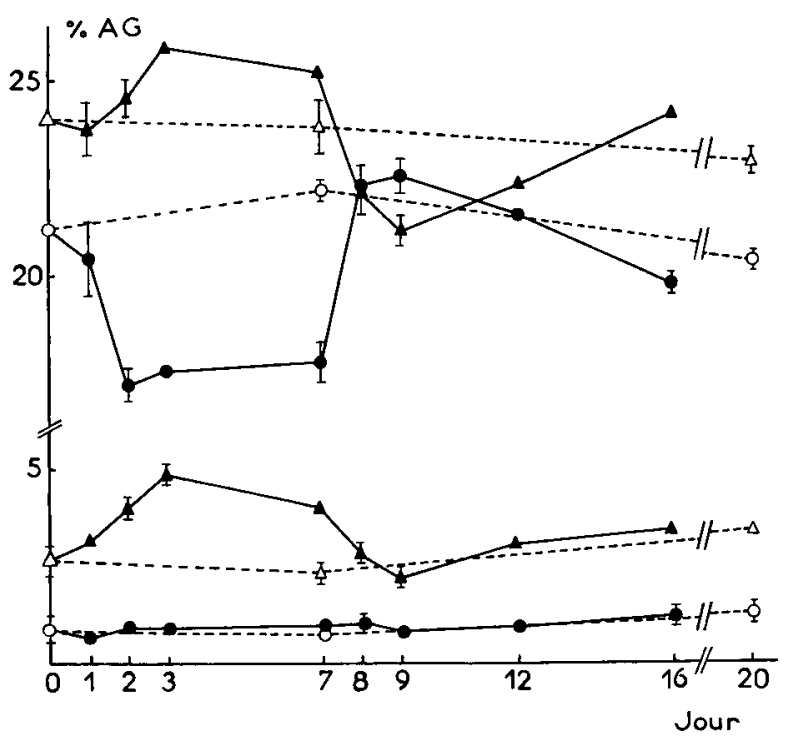

FIG. 7. - Evolution, au cours du temps, de la teneur en acides 16:0 ( $\mathbf{\Delta}$ - $)$ et 18:0 (-1) des CL (courbes du bas) et autres phospholipides (courbes du haut) de mitochondries de foie de rats. Les symboles blancs de même forme correspondent aux mêmes acides gras déterminés chez des rats nourris avec un régime équilibré. Conditions expérimentales : voir légende de la figure 1. 
Acides gras polyinsaturés de la série (n-3).

Les acides gras de la série ( $n-3)$ sont des composés mineurs dans les CL. Pour le plus abondant, l'acide $22: 6(n-3)$, un taux moyen de $1 \%$ environ a été relevé chez les rats du groupe témoin entre les temps 0 et 20 jours. Chez les rats du groupe TRL et chez ceux nourris avec le régime de réplétion, des taux similaires ont été notés. Mais du fait de l'élution tardive de cet acide gras dans nos conditions de CPG, et de sa faible concentration, l'imprécision sur les mesures est grande, et il n'est pas possible de déceler d'évolution dans les différents régimes.

En revanche, l'acide 22:6(n-3) est un acide gras majeur dans les PLm : il représente $15 \%$ de leurs acides totaux au jour 0 (fig. 8). Le passage sur régime TRL provoque une baisse rapide pendant les deux premiers jours, puis plus lente jusqu'au septième jour, où le taux d'acide $22: 6(n-3)$ est de $7,5 \%$. Au temps 66 jours, cet acide gras ne représente plus que $2,5 \%$ des acides gras totaux des PLm. Le retour à la normale s'effectue en 24 à $48 \mathrm{~h}$ après passage au régime de réplétion.

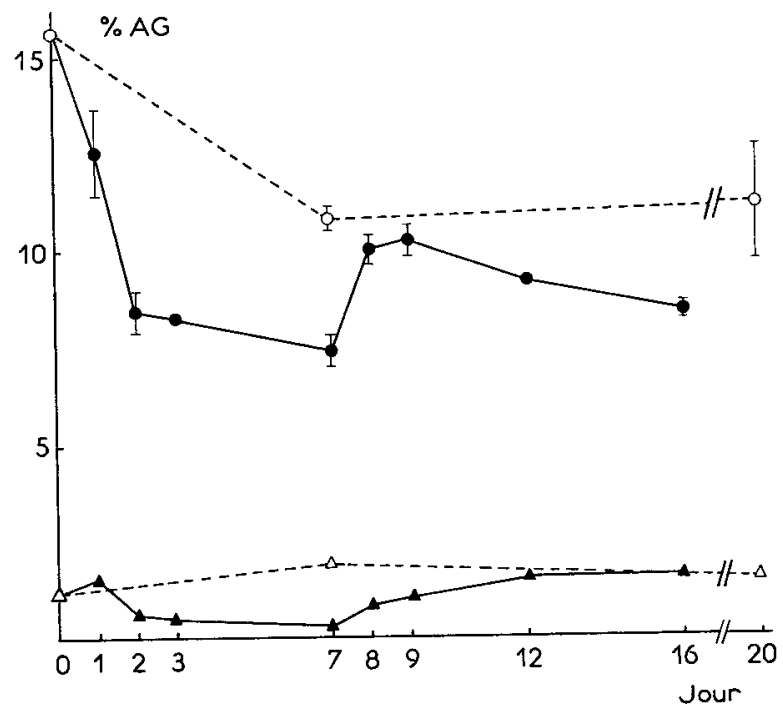

FIG. 8. - Evolution, au cours du temps, des teneurs en acides $22: 5(n-3)(\mathbf{\Delta}-\mathbf{\Delta})$ et $22: 6$ (n-3) (-—) des phospholipides (autres que les $\mathrm{CL}$ ) de mitochondries de foie de rats. Conditions expérimentales : voir légende de la figure 1.

\section{Composés mineurs partiellement caractérisés.}

Dans la figure 9 sont rassemblés les chromatogrammes partiels des EM d'acides gras des PLm à différents temps du régime. Ils illustrent la résolution obtenue dans nos conditions expérimentales entre les acides $20: 2(n-6), 20: 3$ $(n-9)$ et $20: 3(n-6)$ (respectivement, composés 1, 2 et 5 ) et mettent en évidence 

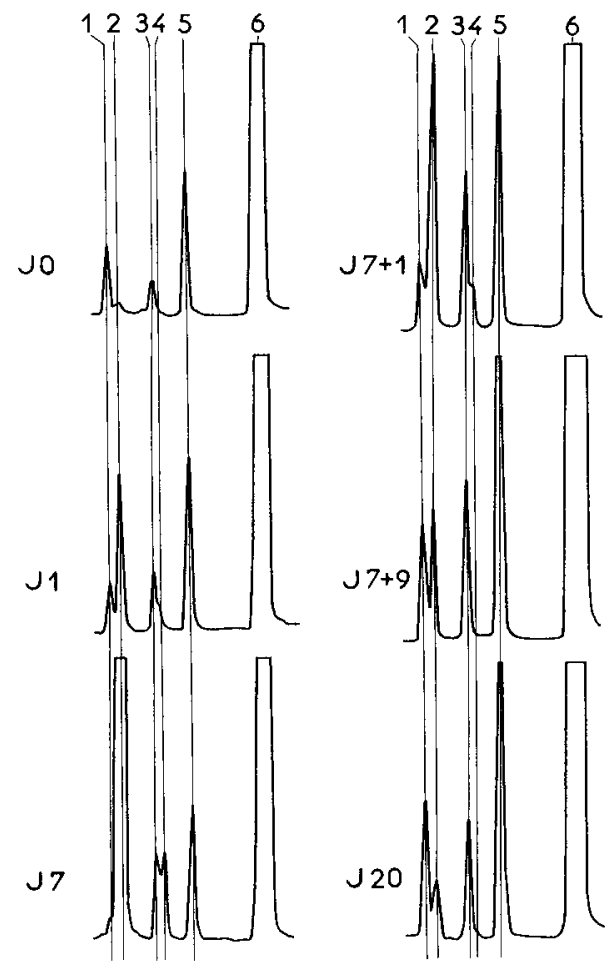

FIG. 9. - Parties de chromatogrammes obtenus par CPG des EM d'acides gras de phospholipides (autres que les $\mathrm{CL}$ ) de mitochondries de foie de rats à différents moments des régimes expérimentaux. $\mathrm{J} 0$ : rats nourris 1 jour avec un régime équilibré; $\mathrm{J} 1$ et $\mathrm{J} 7$ : rats nourris 1 et 7 jours avec un régime à teneur réduite en matière grasse; $J 7+1$ et $J 7+9$ : rats nourris 1 et 9 jours avec un régime équilibré après 7 jours de régime à teneur réduite en matière grasse ; $\mathrm{J} 20$ : rats nourris 20 jours avec un régime équilibré. Pic 1 : acide $20: 2(n-6) ;$ pic $2:$ acide $20: 3(n-9)$; pic 3 : acide $20: 35,11,14$; pic $4:$ acide $20: 3(n-7)$; pic $5:$ acide $20: 3(n-6)$; pic $6:$ acide $20: 4(n-6)$. Les quantités d'EM injectées ne sont pas identiques dans tous les cas.

l'existence de deux composés dont l'un est présent à tous les moments des différents régimes (composé 3), l'autre n'apparaissant qu'en carence (composé 4). Le composé 3 a même temps de rétention en CPG que l'acide $20: 35,11,14$ de l'huile de graine d'if (Madrigal et Smith, 1975) ou préparé par réduction partielle de l'acide arachidonique en présence d'hydrate d'hydrazine (résultats non publiés). II migre sur plaque de silicagel imprégné de nitrate d'argent entre les triènes et les tétraènes, ce qu'on peut attendre d'un triène contenant des doubles liaisons séparées par plus d'un méthylène (De Jong et Van der Wel, 1964). Le composé 4 disparaît lorsque les rats sont à nouveau nourris avec un régime équilibré et il est absent des PLm de rats contrôles. Sur plaque au nitrate d'argent, il migre avec les triènes. La position des doubles liaisons n'a pas été déterminée, mais on peut présumer, de par son élution en CPG entre les acides $20: 3(n-9)$ et (n-6), qu'il s'agit d'un eicosatriène de la série (n-7) ou de la série 
$(n-8)$. Seule la série $(n-7)$ a une réalité biochimique, et il est probable que le composé 4 est l'acide $20: 3(n-7)$, qui s'accumule dans les lipides de rats nourris avec un régime lipidoprive (Sand et al., 1965). Ces acides gras restent mineurs, mais leur somme est voisine du taux d'acide $20: 3(n-6)$ et représente de 0,6 à $0,8 \%$ lors de l'instauration de la déficience. Sur régime de réplétion, l'acide $20: 3$ $(n-7)$ disparaît dès le deuxième jour; l'acide $20: 35,11,14$ représente alors environ $0,3 \%$ des acides gras totaux des PLm, comme chez les rats témoin. Ces deux acides gras inhabituels sont absents des $C L$ des rats des groupes TRL et témoin.

\section{Discussion.}

\section{Modifications des acides gras dans les CL.}

Les modifications en acides gras sont plus lentes à s'établir dans les CL que dans les PLm, tant chez les rats du lot TRL que chez ceux nourris avec le régime de réplétion. Pour ces derniers, nos données concordent avec celles de Bartley (1964) : des CL de foie de rats préalablement nourris avec un régime lipidoprive retrouvent un taux normal d'acide linoléique 10 jours après passage des animaux à un régime contenant de l'huile de maïs. En revanche, dans une étude (Biran et al., 1964) où le taux d'acide linoléique dans les $C L$ de foie était de $9,8 \%$, le transfert des rats sur régime supplémenté en huile de maïs n'a permis d'atteindre en 16 jours que $24,8 \%$, c'est-à-dire le tiers du taux observé dans les CL de rats témoins. Bartley (1964) a également indiqué qu'une baisse de moitié du taux d'acide linoléique dans les $C L$ de rats nourris avec un régime lipidoprive nécessite 6 semaines. Mais la majorité des auteurs constate une chute plus importante de l'acide linoléique en seulement 7-8 jours de régime lipidoprive (Tischer et Glenn, 1964 ; Stancliff et al., 1969). Pour des durées de 1 à 10 semaines, les teneurs finales en acide linoléique sont d'ordinaire de 20 à $30 \%$ (Yamamoto et al., 1965 ; Yurkowski et Walker, 1970 ; Wood, 1975 ; Ishinaga et al., 1982 ; Wolff et al., 1985 a), ou rarement moins (Tischer et Glenn, 1965 ; Stancliff et al., 1969 ; Divakaran et Venkataraman, 1977), sauf si le régime est prolongé (Biran et al., 1964, 1965 ; Johnson et Ito, 1965).

Dans toutes ces études, l'acide linoléique est principalement remplacé par les acides palmitoléique et oléique. La désignation « oléique " recouvre un mélange de deux isomères, l'acide oléique proprement dit [18:1 (n-9)], et l'acide cis-vaccénique [18:1(n-7)], qu'aucune des études citées ne mentionnait. Les proportions d'acides oléique et palmitoléique augmentent toutes deux à peu près à la même vitesse pendant l'instauration de la déficience, et plus rapidement que celle de l'acide $c i s$-vaccénique. Ceci peut s'expliquer en partie par le fait que ce dernier acide gras n'est estérifié qu'aux positions $2\left(2^{\prime \prime}\right)$ des $C L$, alors que les deux autres monoènes sont présents aux positions $1\left(1^{\prime \prime}\right)$ et $2\left(2^{\prime \prime}\right)$ (Wolff et al., $1985 \mathrm{a}$, b). Il est également possible que les enzymes qui contrôlent la composition en acides gras des $C L$ aient une plus grande affinité pour les acides ayant une double liaison en position 9 que pour ceux en ayant une en position 11. D'autre part, les 
proportions des différents monoènes dans le pool d'acides gras accessibles interviennent probablement aussi. Ainsi, les triglycérides du tissu adipeux des rats au temps 66 jours du régime TRL contiennent $37 \%$ d'acide $18: 1$ (n-9), $15 \%$ d'acide 16:1 (n-7), et seulement 5-6\% d'acide 18:1 (n-7) (résultats non montrés).

Dans les études antérieures sur les effets d'une déficience en acide linoléique sur les CL, une augmentation (ou l'apparition) d'acide eicosatriénoïque était mentionnée, mais sa structure n'était pas précisée. Nos résultats montrent qu'il s'agit de l'acide $20: 3(n-6)$, et non de l'isomère (n-9), que nos conditions de CPG permettent de ne pas confondre (fig. 9). De plus, à notre connaissance, aucune étude ne fait mention de l'apparition, dans les $C L$ de rats élevés sur régime lipidoprive, d'un acide octadécadiénoïque différent de l'acide linoléique, l'acide $18: 2(n-7)$. Toutefois, cet isomère reste un constituant mineur des CL et des PLm. Les résultats concernant les PLm confirment ceux de Lemarchal et Munsch (1965), qui ont observé que l'acide $18: 2$ (n-7) n'atteint que 2,6\% des acides gras des phospholipides hépatiques de rats après 3 mois de régime lipidoprive.

Une augmentation significative et réversible du taux d'acide palmitique est observée dans les CL des rats du groupe TRL. II semble donc que le taux d'acide palmitique soit variable dans les CL de foie, tout comme il varie dans les CL d'un organe à l'autre (Wolff et al., 1985 b), mais dans une marge étroite. La faible teneur en acides gras saturés des $C L$ est actuellement inexpliquée : les précurseurs des CL — phosphatidylglycérol et CDP-diglycéride - contiennent de tels acides gras (Soula et Champanet, 1973; Thompson et MacDonald, 1976) et les précurseurs saturés sont des substrats au moins aussi bons que leurs homologues insaturés pour la synthèse des CL in vitro (Hostetler et al., 1975).

\section{Modifications des acides gras dans les PLm.}

Dans les PLm, les acides $18: 2(n-6)$ et $20: 4(n-6)$ diminuent rapidement pendant les 3 premiers jours sur régime TRL, puis se stabilisent pendant les 4 jours suivants. Il en est de même pour l'acide 22:6 (n-3). Ces baisses d'acides polyinsaturés sont, comme dans les $\mathrm{CL}$, compensées principalement par une augmentation des acides monoénoïques, surtout par celle de l'acide oléique, mais aussi par l'apparition d'acide $20: 3(n-9)$. Les monoènes représentent $6,4 \%$ des acides gras totaux des PLm au temps 0 des régimes, $22,1 \%$ à 3 jours, $22,3 \%$ à 7 jours et $19,5 \%$ à 66 jours. Toutes les études de déficience en acide linoléique à long terme ont mis en évidence des modifications similaires. Mais, en ce qui concerne les événements précoces, les rares travaux réalisés (Peluffo et al., 1976 ; Brenner et al., 1981 ; Flick, 1981) ont conduit à des résultats discordants. Notre étude montre que la baisse d'acides polyinsaturés des 3 premiers jours $(-19 \%)$ est surtout compensée par une augmentation des monoènes $(+15,9 \%)$, l'acide $20: 3(n-9)$ ne contribuant à leur remplacement que pour une faible part $(3,6 \%)$. Ce n'est que par la suite, sur des périodes longues, qu'il se substitue aux acides polyinsaturés. Au temps 66 jours, où ceux-ci ont subi une baisse supplémentaire de $6 \%$, l'acide $20: 3(n-9)$ a augmenté de près de $10 \%$, les monoènes étant, eux, restés relativement stables. 
Une augmentation de la proportion d'acide oléique dans les acides octadécénoïques a été observée antérieurement dans les phospholipides totaux de cœur et de reins de rats nourris avec un régime lipidoprive (Spence, 1971). Nos observations montrent que ces variations sont plus importantes dans les CL que dans les PLm de mitochondries de foie. Si la signification biologique de ces changements n'est pas connue, il n'est pas inutile de rappeler que chez le rat adulte, c'est l'acide cis-vaccénique qui est l'acide octadécénoïque majeur des phospholipides du foie (Wood, 1979).

L'acide $20: 35,11,14$ a été détecté dans les lipides du foie de rats nourris 2 mois avec un régime lipidoprive (Schmitz et al., 1977). D'un autre côté, Ullman et Sprecher (1971) ont montré que l'acide $20: 2(n-6)$, dans certaines conditions, est désaturé en acide $20: 35,11,14$. Nos observations suggèrent que cet acide gras est présent naturellement dans les PLm des mitochondries du foie de rat, mais pas dans les $C L$, que les animaux soient élevés sur régime $T R L$ ou sur régime équilibré.

L'acide $20: 3(n-7)$ apparaît en faible quantité dans les lipides totaux de différents organes de rats nourris avec un régime lipidoprive pendant de longues périodes (Klenk et Tschöpe, 1963; Sprecher, 1971 ; Spence, 1971). Au temps 66 jours du régime TRL, nous avons observé que les phospholipides totaux des mitochondries de foie contiennent $0,7 \%$ de ce triène de la série palmitoléique, mais que les $C L$ en sont dépourvus.

\section{Vitesses des modifications de compositions.}

Les modifications des compositions en acides gras peuvent se faire selon deux voies principales : synthèse de novo et renouvellement des acides gras par désacylation-réacylation. Les expérimentations faites in vitro et in vivo ont indiqué que ces deux mécanismes opéraient plus rapidement dans le cas des phospholipides diacylés que dans le cas des CL (Bard et al., 1972; Soula et Champanet, 1973; Landriscina et al., 1976). Nos observations sont en accord avec ces résultats et suggèrent qu'un mécanisme de régulation visant à limiter la perte en acides polyinsaturés dans les PLm se met en place rapidement lorsque les rats sont nourris avec le régime TRL. Les CL ne sont apparemment pas sujets à une telle régulation. De plus, le retour à la normale est nettement plus long à se faire dans les $C L$ que dans les $P L m$ après passage au régime équilibré.

Dans les $C L$, l'acide linoléique est présent à la fois aux positions $1\left(1^{\text {") }}\right.$ et aux positions $2\left(2^{\prime \prime}\right)$, avec une préférence marquée pour les positions $1\left(1^{\prime \prime}\right)$ (Wolff et al., $1985 \mathrm{~b}$, et références citées). Bien que l'on sache que la durée de demi-vie de l'acide linoléique dans les CL est plus longue que dans les PLm (Landriscina et al., 1976), rien n'est connu des différences éventuelles entre les positions 1 (1") et 2(2"), à l'exception de l'observation de Bard et al. (1972) qui ont montré que seuls les acides gras des positions $1(1 ")$ peuvent être remplacés par de l'acide linoléique in vitro. A priori, il y a peu de raisons de penser que les vitesses de désacylation-réacylation soient identiques aux différentes positions des CL. Une étude de la distribution positionnelle des acides gras des CL hépatiques de rats nourris avec un régime lipidoprive pendant 12 jours (Wolff et al., 1985 a) a 
montré que la baisse d'acide linoléique est sensiblement plus importante aux positions 2(2") qu'aux positions $1\left(1^{\prime \prime}\right)$. D'autre part, les $C L$ d'organes divers du rat, dont les teneurs en acide linoléique varient entre 78,4 et $61,8 \%$, ont des taux voisins d'acide linoléique aux positions 1 (1") (de 89,3 à $85 \%$ ), mais variables aux positions 2(2") (de 67,8 à 37,8\%) (Wolff et al., 1985 a). Si la teneur en acide linoléique dans les $C L$ est liée à la disponibilité de cet acide dans les organes, on peut envisager qu'une baisse d'acide linoléique consécutive à un changement alimentaire se traduira par une baisse plus rapide de cet acide gras aux positions 2(2") qu'aux positions $1\left(1^{\prime \prime}\right)$.

\section{Caractéristiques structurales des acides gras des $C L$.}

On peut observer que la majorité des acides gras des $C L$ possède une double liaison en position 9 ou 11 (ou, au minimum, en position 8 chez les rats du groupe TRL). Les acides qui ont une double liaison plus proche du groupe carboxyle sont quantitativement mineurs dans les $C L$, mais abondants dans les PLm. C'est le cas des acides $20: 4(n-6)$ et $22: 6(n-3)$, et, chez les rats du groupe TRL, de l'acide $20: 3(n-9)$. Les CL peuvent également renfermer, chez divers animaux, d'autres acides gras ayant leur première double liaison en position 9 ou 11 : acide $\alpha$-linolénique $(18: 39,12,15)$ (pour se limiter aux mammifères: MacFarlane, 1961 ; Fleischer et Rouser, 1965 ; Yamamoto et al., 1965) ou acide $20: 311,14$, 17 (Seher et al., 1983). Lorsqu'ils sont présents, ces acides gras sont estérifiés dans les CL à des concentrations qui sont supérieures à celles présentées par les autres phospholipides de même origine. Une exception majeure est représentée par les CL du cerveau, qui contiennent $20 \%$ d'acide arachidonique et $13-15 \%$ d'acide docosahéxaénoïque (Yamaoka et al., 1988).

L'affinité des CL pour les acides gras à première double liaison en position 9 ou 11 nous semble être la particularité, plus que celle souvent retenue de leur forte teneur en acide linoléique, qui caractérise plus généralement et plus précisément les CL. Dès lors, on peut s'interroger sur l'importance de ce paramètre structural dans la fonctionnalité des CL et se demander quelle peut être l'influence du nombre de doubles liaisons sur la fonction des CL, dans la mesure où la position de la première double liaison des acides gras est respectée. En effet, on peut concevoir qu'il existe une zone structurée et cohésive entre les portions d'acides gras situées entre les groupes carboxyles et les doubles liaisons en position 9 ou 11 , et, au-delà de celles-ci, une zone moins ordonnée, plus «fluide». Selon l'importance des deux zones sur la fonctionnalité des CL, une modification du nombre de doubles liaisons pourrait ne pas avoir d'effets physiologiques si ces doubles liaisons sont présentes au-delà de la position 9. Inversement, on peut se demander quelle peut être l'influence de la présence d'acides gras, tels que les acides $20: 4(n-6)$ ou $22: 6(n-3)$, ayant des doubles liaisons dans cette zone, sur la fonctionnalité des CL.

L'étude de l'influence des matières grasses alimentaires sur la fonction physiologique spécifique des CL est délicate, car toute manipulation visant à en modifier les acides gras affecte également les autres phospholipides. Toutefois, dans le modèle expérimental proposé, il existe une période, entre les jours 1 - 2 et 
9 du régime de réplétion, où seuls les CL ont une composition en acides gras modifiée par rapport au groupe témoin. Cette période pourrait donc être mise à profit pour tenter de rechercher d'éventuelles altérations fonctionnelles d'enzymes mitochondriales, altérations qui pourraient alors être associées aux CL.

Reçu en mars 1988

Accepté en juillet 1988

Remerciements. - L'auteur remercie Mlle Laurence Surlanne pour son aide technique, Mme Nicole Combe et M. le Professeur B. Entressangles pour l'intérêt qu'ils ont porté à la réalisation de ce travail, et Mme Isabelle Wolff-Augarde qui a assuré gracieusement le traitement informatique du texte.

\section{References}

ASTORG P.-O., CHEVALIER J., 1987. Polyunsaturated fatty acids in tissues of rats fed trielaidin and high or low levels of linolenic acid. Lipids, 22, 1025-1030.

BARD D., COLARD O., BEREZIAT G., 1972. Incorporation in vitro du ${ }^{14} \mathrm{C}$ linoléate dans le diphosphatidylglycérol des membranes internes des mitochondries de foie de rat. C.R. Acad. Sci. Paris, 275, 2429-2431.

BARTLEY W., 1964. Lipids of intracellular organelles, 369-381. In DAWSON R. M. C., RHODES D. N., Metabolism and physiological significance of lipids, John Wiley and Sons, New York.

BEYER K., KLINGENBERG M., 1985. ADP/ATP carrier protein from beef heart mitochondria has high amounts of tightly bound cardiolipin, as revealed by ${ }^{31} \mathrm{P}$ nuclear magnetic resonance. Biochemistry, 24, $3821-3826$.

BIRAN L. A., BARTLEY W., CARTER C. W., RENSHAW A., 1964. Studies on essential fatty acid deficiency. Effect of the deficiency on the lipids in various rat tissues and the influence of dietary supplementation with essential fatty acids on deficient rats. Biochem. J., 93, 492-498.

BIRAN L. A., BARTLEY W., CARTER C. W., RENSHAW A., 1965. Studies on essential fatty acid deficiency. Effect of the deficiency on the lipids in liver mitochondria and oxidative phosphorylation. Biochem. J., 94, 247-251.

BLOMSTRAND R., SVENSSON L., 1983. The effects of partially hydrogenated marine oils on the mitochondrial function and membrane phospholipid fatty acids in rat heart. Lipids, 18 , $151-170$.

BRENNER R. R., GARDA H., DE GOMEZ DUMM I. N. T., PEZZANO H., 1981. Early effects of EFA deficiency on the structure and enzymatic activity of rat liver microsomes. Progr. Lipid Res., 20, 315-321.

BRUCE A., 1974. Changes in the concentration and fatty acid composition of phospholipids in rat skeletal muscle during postnatal development. Acta physiol. scand., 90, 743-749.

COLBEAU A., NACHBAUR J., VIGNAIS P. M., 1971. Enzymic characterization and lipid composition of rat subcellular membranes. Biochim. Biophys. Acta, 249, 462-492.

COMBE N., RIETSCH J., WOLFF R., ENTRESSANGLES B., 1980. Implications de l'incorporation d'isomères trans d'acides gras insaturés au niveau des membranes cellulaires. Ann. Nutr. Alim. 34, 305-316.

DE JONG K., VAN DER WEL H., 1964. Identification of some iso-linoleic acids occuring in butter fat. Nature, 202, 553-555.

DIVAKARAN P., VENKATARAMAN A., 1977. Effect of dietary fats on oxidative phosphorylation and fatty acid profile of rat liver mitochondria. J. Nutr., 107, 1621-1631.

FLEISCHER S., ROUSER G., 1965. Lipids of subcellular particles. J. Am. Oil Chem. Soc., 42, 588-607. 
FLICK P. K., 1981. Rapid changes in rat liver fatty acid composition during fat-free and polyunsaturated fat feeding. Nutr. Rep. Int., 24, 667-674.

FOLCH J., LEES M., SLOANE-STANLEY G. H., 1957. A simple method for the isolation and purification of total lipides from animal tissues. J. biol. Chem., 226, 497-509.

FRY M., BLONDIN G. A., GREEN D. E., 1980. The localization of tightly bound cardiolipin in cytochrome oxidase. J. biol. Chem., 255, 9967-9970.

FRY M., GREEN D. E., 1981. Cardiolipin requirement for electron transfer in complex I and III of the mitochondrial respiratory chain. J. biol. Chem., 256, 1874-1880.

GETZ G. S., BARTLEY W., 1959. A cardiolipin-like compound in rat liver mitochondria. Nature, 184, $1229-1230$

HOSTETLER K. Y., GALESLOOT J. M., BOER P., VAN DEN BOSCH H., 1975. Further studies on the formation of cardiolipin and phosphatidylglycerol in rat liver mitochondria. Effect of divalent cations and the fatty acid composition of CDP-diglyceride. Biochim. Biophys. Acta, 380, 382-389.

IOANNOU P. V., GOLDING B. T., 1979. Cardiolipins : their chemistry and biochemistry. Prog. Lipid Res., 17, 279-318.

ISHINAGA M., SATO J., KITAGAWA Y., SUGIMOTO E., KITO M., 1982. Perturbation of phospholipid metabolism by erucic acid in male Sprague-Dawley rat heart. J. Biochem. Tokyo, 92, 253-263.

JOHNSON R. M., ITO T., 1965. Effects of a nutritional deficiency of unsaturated fats on the distribution of fatty acids in rat liver mitochondrial phospholipids. J. Lipid Res., 6, 75-79.

KLENK E., TSCHÖPE G., 1963. Über die Natur der in den Leberlipoiden von fettfrei ernährten Ratten vorkommenden ungesättigten Fettsäuren. Z. Physiol. Chem., 334, 193-200.

LANDRISCINA C., MEGLI F. M., QUAGLIARIELLO E., 1976. Turnover of fatty acids in rat liver cardiolipin : comparison with other mitochondrial phospholipids. Lipids, 11, 61-66.

LEMARCHAL P., MUNSCH N., 1965. Etude sur le métabolisme de l'acide élaïdique chez des rats carencés en acides gras indispensables. C.R. Acad. Sci. Paris, 260, 714-716.

MacFARLANE M. G., 1961. Cardiolipin and other phospholipids in ox liver. Biochem. J., 78, 44-51.

MADRIGAL R. V., SMITH C. R. Jr., 1975. Taxus baccata seed oil : a new source of cis-5,cis-9octadecenoic acid. Lipids, 10, 502-504.

McMURRAY W. C., JARVIS E. C., 1979. Partial purification of diphosphatidylglycerol synthetase from liver mitochondrial membranes. Can. J. Biochem., 58, 771-776.

MERLE P., KADENBACH B., 1982. Kinetic and structural differences between cytochrome $c$ oxidases from beef liver and heart. Eur. J. Biochem., 125, 239-244.

MORRISON W. R., SMITH L. M., 1964. Preparation of fatty acid methyl esters and dimethylacetals from lipids with boron fluoride-methanol. J. Lipid Res., 5, 600-608.

PALMER J. W., SCHMID P. C., PFEIFER D. R., SCHMID H. H. O., 1981. Lipids and lipolytic enzyme activities of rat heart mitochondria. Arch. Biochem. Biophys., 211, 674-682.

PATTON G. M., FASULO J. M., ROBINS S. J., 1982. Separation of phospholipids and individual molecular species of phospholipids by high-performance liquid chromatography. J. Lipid Res., 23, 190-196.

PELUFFO R. O., NERVI A. M., BRENNER R. R., 1976. Linoleic acid desaturation activity of liver microsomes of essential fatty acid deficient and sufficient rats. Biochim. Biophys. Acta, 441, $25-31$.

SAND D., SEN N., SCHLENK H., 1965. Positional isomerism of unsaturated fatty acids in the rat. Quantification of isomeric mixtures. J. Am. Oil Chem. Soc., 42, 511-516.

SANTIAGO E., LOPEZ-MORATALLA N., SEGOVIA J. L., 1973. Correlation between losses of mitochondrial ATPase activity and cardiolipin degradation. Biochem. Biophys. Res. Com., 53, 439-445.

SCHMITZ B., MURAWSKI U., PFLÜGER M., EGGE H., 1977. Positional isomers of unsaturated fatty acids in rat liver lipids. Lipids, 12, 307-313.

SEHER A., SPIEGEL H., KÖNKER S., OSLAGE H. J., 1983. Ernährungsphysiologische Wirkung unterschiedlicher Gemische von Öl-, Linol- und Linolensäure bei wachsenden Schweinen. 3. Wirkung auf die Nierenlipide. Fette Seifen Anstrichm., 85, 295-304. 
SOULA G., CHAMPANET C., 1973. Analyse et métabolisme des phospholipides du cœur. Incorporation in vivo de ${ }^{32} \mathrm{P}$ orthophosphate dans les lipides cardiaques du rat. Biochimie, 55, $1229-1306$.

SPENCE M. W., 1971. The effect of a fat-free diet on esterified monoenoic fatty acid in rat tissues. Lipids, 6. $831-835$.

SPRECHER H. W., 1971. The total synthesis and metabolism of octadeca-5,8,11-trienoate, eicosa-10,13-dienoate and eicosa-7,10,13-trienoate in the fat-deficient rat. Biochim. Biophys. Acta, 231, 122-130.

STANCLIFF R. C., WILLIAMS M. A., UTSUMI K., PACKER L., 1969. Essential fatty acid deficiency and mitochondrial function. Arch. Biochem. Biophys., 131, 629-642.

THOMPSON W., MacDONALD G., 1976. Cytidine diphosphate diglyceride of bovine brain. Positional distribution of fatty acids and analysis of major molecular species. Eur. J. Biochem., 65, 107-111.

TISCHER K., GLENN J. L., 1965. The fatty acids of individual liver phosphatides from rats grown on a fat-free diet. Biochim. Biophys. Acta, 98, 502-511.

ULLMAN O., SPRECHER H., 1971. An in vitro and in vivo study of the conversion of eicosa-5,11,14trienoic acid and of the conversion of eicosa-11-enoic acid in the rat. Biochim. Biophys. Acta, 248, 186-197.

VAN DEN BOSCH H., 1974. Phosphoglyceride metabolism. Annu. Rev. Biochem., 43, 243-277.

WOLFF R. L., COMBE N., ENTRESSANGLES B., 1984. Incorporation et élimination de l'acide élaïdique dans les différents phospholipides des mitochondries ou de leurs membranes chez le rat. Influence de l'organe et de l'âge. Rev. Fr. Corps Gras, 31, 161-170.

WOLFF R. L., COMBE N. A., ENTRESSANGLES B., 1985 a. Cardiolipin structure: effect of a fat-free diet on the fatty acid positional distribution. Rev. Fr. Corps Gras, 31, 295-299.

WOLFF R. L., COMBE N. A., ENTRESSANGLES B., 1985 b. Positional distribution of fatty acids in cardiolipin from 21 -day-old rats. Lipids, 20, 908-914.

WOOD R., 1975. Hepatoma, host liver, and normal rat liver phospholipids as affected by diet. Lipids, 10. $736-745$.

WOOD R., 1979. Distribution of dietary geometrical isomers in brain, heart, kidney, liver, lung, muscle, spleen, adipose and hepatoma, 213-281. In EMKEN E. A. and DUTTON H. J., Geometrical and positional fatty acid isomers, The American Oil Chemists' Society, Champaign, Illinois.

WOOD R., UPRETI G. C., DE ANTUENO R. J., 1986. A comparison of lipids from liver and hepatoma subcellular membranes. Lipids, 21, 292-300.

YAMAMOTO A., ISOZAKI M., HIRAYAMA K., SAKAI Y., 1965. Influence of dietary fatty acids on phospholipid fatty acid composition in subcellular particles of rat liver. J. Lipid Res., 6, 295-300.

YAMAOKA S., URADE R., KITO M., 1988. Mitochondrial function in rats is affected by modification of membrane phospholipids with dietary sardine oils. J. Nutr., 118, 290-296.

YURKOWSKI M., WALKER B. L., 1970. Lipids of the intestinal mucosa of normal and essential fatty acid deficient rats. Can. J. Physiol. Pharmacol., 48, $631-639$. 